\title{
Obstructive Sleep Apnea and Metabolic Syndrome: Is there A Correlation?
}

\author{
Adelina Anton $^{1 *}$, Doina Ecaterina Tofolean ${ }^{1,2}$, Petronela Ariadna Fildan ${ }^{1,3}$, Elena Dantes ${ }^{1,3}$ and Oana \\ Cristina Arghir 1,3 \\ ${ }^{1}$ Faculty of Medicine, "Ovidius" University, Romania \\ ${ }^{2}$ Department of Pneumology, County Emergency Clinical Hospital, Romania
}

${ }^{3}$ Department of Pneumology, Clinical Pneumophtisiology Hospital, Romania

*Corresponding author: Adelina Anton, Faculty of Medicine, “Ovidius” University, Romania

\begin{tabular}{|c|}
\hline ARTICLE INFO \\
\hline Received: 幽 January 13, 2020 \\
\hline Published: 崖 January 23, 2020 \\
\hline
\end{tabular}

Citation: Doina Ecaterina Tofolean, Adelina Anton, Petronela Ariadna Fildan, Elena Dantes, Oana Cristina Arghir. Obstructive Sleep Apnea and Metabolic Syndrome: Is there A Correlation?. Biomed J Sci \& Tech Res 24(5)-2020. BJSTR. MS.ID.00417.

Keywords: Obstructive Sleep Apnea; Metabolic Syndrome; Obesity; CardioMetabolic Risk Factor

Abbreviations: OSA: Obstructive Sleep Apnea; MS: Metabolic Syndrome; AHA: American Heart Association; AHI: ApneaHypopnea Index; BMI: Body Mass Index; NC: Neck Circumference; NHLBI: National Heart, Lung, and Blood Institute

\section{ABSTRACT}

Introduction: Obstructive Sleep Apnea (OSA) and Metabolic Syndrome (MS) are common disorders with an escalating prevalence and systemic consequences. OSA is defined as recurrent episodes of complete or partial upper airway closure during sleeping. Metabolic syndrome comprises a heterogeneous group of changes including diabetes, dyslipidemia, obesity, hypertension.

The aim of this study was to evaluate the association between OSA and MS.

Methods: We conducted a prospective study of patients who underwent seep study in Constanta Sleep Disorders Center, between 2015-2019. The patient group consisted of 151 individuals (103 male and 48 females). All patients underwent polygraphy, blood sampling and measurement of anthropometric variables. OSA was consider present when AHI $>15$. MS was defined according to the National Heart, Lung, and Blood Institute (NHLBI) and the American Heart Association (AHA) guidelines.

Results: Out of a total of 151 patients with OSA, 90 (59.6\%) were found to have MS. All of the separate components of metabolic syndrome were common in our patients, but the diagnosis was made based on three (57.7\%), four or all criteria $(42.3 \%)$. Furthermore, all the anthropometric variables were associated with MS. The prevalence of MS among OSA patients increased with increasing AHI. Obesity was a strong link to both, in particular visceral obesity for MS and enlargement of soft tissue structure within and surrounding the airway for OSA.

Conclusion: Patients with OSA have a high prevalence of metabolic syndrome. Thus, this interplay could disclose a role for OSA screening in patient with metabolic abnormalities.

\section{Introduction}

Obstructive Sleep Apnea (OSA) represents a common sleep disorder with multisystem involvement, but largely underdiagnosed and undertreat that is characterized by repetitive episodes of apnea and hypopnea during sleep leading to sleep fragmentation and intermittent hypoxia [1]. OSA divert from obstruction of the airway which varies between individuals from obstruction due to abnormal anatomy, obese anatomy (neck distribution of fat) or decrease in neuromuscular tone. It is an increasingly prevalent disease, affecting approximately $4 \%$ of men and $2 \%$ of woman, being linked to the epidemic of obesity, accounting that OSA is diagnosed in approximately $40-70 \%$ of the obese patients [2]. The severity of an individual's sleep disease is defined by ApneaHypopnea Index (AHI). Thus, mild OSA is characterized by 5-15 apnea-hypopnea events per hour, whereas moderate and severe OSA account 15-30 apnea-hypopnea events per hour, respectively $>30$ apnea-hypopnea events per hour. Although the main symptom 
of OSA is excessive daytime sleepiness, the disease is sharing cardiovascular risk factors with Metabolic Syndrome (MS) like obesity, hypertension, insulin-resistance, which predispose individuals to early development of cardiovascular disease [2,3].

Metabolic Syndrome (MS) is characterized by a constellation of different metabolic abnormalities comprising of obesity, dyslipidemia, hypertension, diabetes or insulin resistance which has a substantial increasingly prevalence in last decades, obesity being shown to be the main precursor of it. Metabolic syndrome and OSA share common pathogenic pathways, including alterations in autonomic nervous system regulation, increased inflammatory activity, and alterations in adipokine levels and endothelial dysfunction $[3,4]$. Because of these multiple mutual features, we hypothesis that OSA could be associated with metabolic syndrome and with the overall prevalence of this syndrome, independently of obesity. The purpose of this study was to evaluate the association between OSA, MS and its components and to determine if this association can be used to predict the presence of OSA among patients with metabolic changes and vice versa.

\section{Material and Methods}

We conducted a prospective study of patients who referred to Sleep Disorders Center from County Emergency Clinical Hospital of Constanta, for evaluation of OSA, between January 2015 - June 2019. 207 consecutive individuals were referred for sleep study on the bases of clinical indications identified by physicians in the course of their regular assessment. All patients underwent overnight respiratory polygraphy study, blood sampling (cholesterol profile, fasting glucose) and measurement of anthropometric variables (weight, Body Mass Index (BMI), Neck Circumference (NC), waist circumference), blood pressure measurements. We used patient's medical record to identify the information that included the presence of comorbidities like hypertension, diabetes, dyslipidemia or patient's currently treatments with antihypertensive drugs, insulin or oral hypoglycemic agents, lipid-lowering medication.

OSA was defined according to apnea hypopnea index as follows: no OSA (AHI $<5$ events $/ \mathrm{h}$ ), mild OSA (AHI $\geq 5$ events $/ \mathrm{h}$ but $<15$ events/h), moderate OSA (AHI $\geq 15$ events/h but $<30$ events $/ \mathrm{h}$ ) and severe OSA (AHI $\geq 30$ events/h). We scored apneas as events characterized by decreasing in airflow signal by $80 \%$ or greater with continuing respiratory effort persisting at least 10 seconds. Hypopnea events were scored at $30 \%$ or greater decrease in airflow signal with continued respiratory effort persisting at least 10 seconds associated with a desaturation of at least 4\%. Because we used only overnight respiratory polygraphy study OSA was consider present when AHI $>15$ events/h (which is the cutoff for positive diagnostic in these cases).

The diagnosis of metabolic syndrome was based according to the National Heart, Lung, and Blood Institute (NHLBI) and the American Heart Association (AHA) guidelines, by the presence of at least 3 out of the following 5 criteria: a) Abdominal obesity (waist circumference $\geq 94 \mathrm{~cm}$ in men or $\geq 80 \mathrm{~cm}$ in women),

b) Alow level of high-density lipoprotein cholesterol $(<40$ $\mathrm{mg} / \mathrm{dl}$ in men or $<50 \mathrm{mg} / \mathrm{dl}$ in women) or receiving lipidlowering drug therapy,

c) Elevated triglyceride level ( $\geq 150 \mathrm{mg} / \mathrm{dl}$ ) or receiving drug therapy for hypertriglyceridemia,

d) Elevated blood pressure (systolic $\geq 130 \mathrm{mmHg}$ or diastolic $>85 \mathrm{mmHg}$ ) or receiving drug therapy for hypertension,

e) Fasting plasma glucose level ( $\geq 100 \mathrm{mg} / \mathrm{dl}$ ) or receiving drug therapy for hyperglycemia.

We compared the presence of MS among OSA patients, we assessed the association and correlated it to the AHI severity. Also, we compared the presence of hypertension, dyslipidemia, diabetes among patients that didn't met the criteria for diagnosis of MS. A p value of 0.05 or less was considered to be statistically significant.

\section{Results}

There were 207 patients evaluated in our Sleep Disorders Center during the study period. Out of these individuals, we included in the analysis only the subjects with AHI>15 events/h, summing 151 adults. The group consisted in 103 (68.2\%) males and 48 (31.8\%) females, age range 28-82 years (mean age was 59.01 years), showing that most of the patients were diagnosed in the fifth and sixth decade of life. There were 55 patients (36.4\%) with AHI between 15-30 ev/h and 96 (63.6\%) patients with $\mathrm{AHI}>30 \mathrm{ev} / \mathrm{h}$ (mean AHI $38 \mathrm{ev} / \mathrm{h}$ in men and $24 \mathrm{ev} / \mathrm{h}$ in women), revealing increasing AHI severity over 45 years-old patients. Another important group characteristic was obesity. For an objective evaluation of it we used several parameters like body mass index, neck circumference, waist circumference. Only 30 patients (19.86\%) were found to be overweight or had normal BMI, the rest of 121 patients (80.14\%) presented different degrees of obesity (Table 1). Of the entire group of 151 patients with moderate and severe OSA, 90 patients (59.6\%) were found to have metabolic syndrome and 61 patients (40.4\%) were classified as not having metabolic syndrome and the diagnosis was made based on three (52 patients accounting 57.7\%) , four or all criteria (38 patients accounting 42.3\%). Furthermore, all the anthropometric variables were associated with MS. The prevalence of MS among OSA patients increased with increasing AHI (Table 2).

Table 1: Group characteristics.

\begin{tabular}{|c|c|}
\hline Characteristics & Results* \\
\hline Total group (N=151) & $59.01+/-16.34(28-82)$ \\
\hline Age, $\mathbf{y}$ & $48 / 103$ \\
\hline Female $/$ Male & $30.9+/-5.7(22.5-45.2)$ \\
\hline BMI & 29.8 \\
\hline AHI & \\
\hline
\end{tabular}

Note: *Values are mean +/- SD (range), BMI-Body Mass Index, AHI-Apnea Hypopnea Index. 
Table 2: Characteristics of men and women.

\begin{tabular}{|c|c|c|c|}
\hline Characteristics & Men $\mathbf{( N = 1 0 3 )}$ & Women(N=48) & P \\
\hline Age, $\mathbf{y}$ & 58.03 & 55.09 & \\
\hline BMI & 32.5 & 29.9 & \\
\hline Neck circumference & 43.7 & 39.9 & $<0.001$ \\
\hline Waist circumference & 89.3 & 102.5 & 0.003 \\
\hline AHI & 24 & 38 & \\
\hline MS & 58 & 32 & 0.001 \\
\hline Diabetes & 25 & 23 & \\
\hline Dyslipidemia & 45 & 22 & \\
\hline Hypertension & 77 & 33 & \\
\hline
\end{tabular}

Note: BMI-Body Mass Index, AHI-Apnea Hypopnea Index, MSMetabolic Syndrome.

Obesity was a strong link to both, in particular visceral obesity (increased waist circumference) for MS and enlargement of soft tissue structure within and surrounding the airway (increased neck circumference) for OSA. The mean BMI was $30.9 \mathrm{~kg} / \mathrm{m}^{2}$, mean abdominal circumference was $89.3 \mathrm{~cm}$ in women and $102.5 \mathrm{~cm}$ in men. Waist circumference correlated better with AHI than with the BMI ( $p=0.003$ ) but when assessing obesity, a more statistically significant variable evaluated was neck circumference. Neck circumference correlate with OSA severity $(\mathrm{p}<0.001)$ and we can conclude is a useful marker in clinical practice to assess the need of a sleep study (Table 3). All of the separate components of metabolic syndrome were common in our patients, but we compared the presence of diabetes, hypertension or dyslipidemia between patients that met the definition for OSA with or without MS. Hypertension was significantly more common in the group with MS than in patients without, suggesting that hypertension seems to be the factor most closely associated with the presence of metabolic syndrome in a patient with OSA (Figure 1).

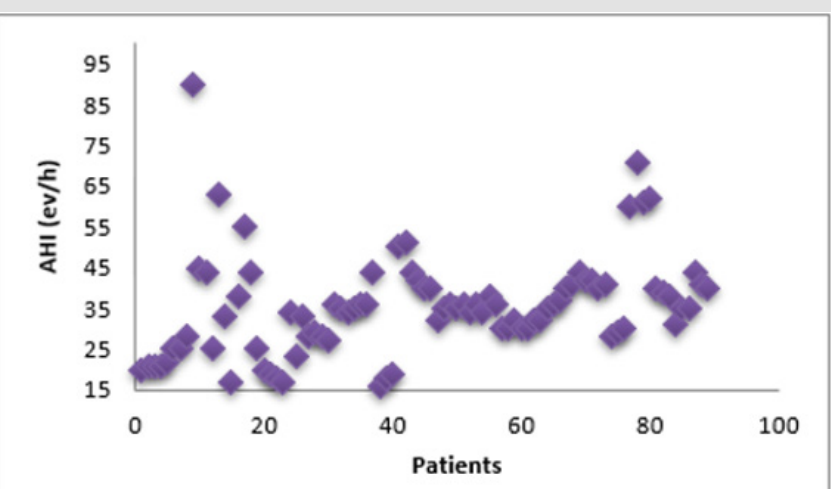

Figure 1: The correlation plot demonstrating strong relationship between the presence of metabolic syndrome and OSA severity.
Table 3: Prevalence of MS and its components and correlation to OSA severity.

\begin{tabular}{|c|c|c|c|}
\hline & AHI 15-29 ev/h & AHI $>\mathbf{3 0} \mathbf{~ e v} / \mathbf{h}$ & P \\
\hline MS & $(\mathrm{N}=55)$ & $(\mathrm{N}=96)$ & \\
\hline Diabetes & 40 & 50 & 0.004 \\
\hline Hypertension & 20 & 28 & 0.3 \\
\hline Dyslipidemia & 48 & 52 & 0.001 \\
\hline
\end{tabular}

Note: Values are number, AHI-Apnea Hypopnea Index, MSMetabolic Syndrome.

\section{Discussion}

This study assessed the association of OSA with MS and different parameters of it. Metabolic syndrome was common in patients with OSA because obesity was a shared risk factor. Analysis of metabolic syndrome component variables shows that hypertension is the primary variable associated with a diagnosis of OSA. Overall, the prevalence of metabolic syndrome appears to be related to the severity of apnea-hyponea index. These results support the concept that the number of important features of MS increases with the increase in OSA severity, regardless of the BMI. OSA and MS are significant cardiovascular risk factors that act synergistically. Furthermore, the study grant us to conclude that OSA is related with increasing cardio-metabolic morbidity. In addition, recognition and treatment of both, OSA and MS may decrease the cardiovascular risk in these types of patients. In addition, MS can exacerbate OSA creating a bidirectional relationship between these two pathologies. The presence of MS may be the trigger to the development of OSA. Thus, this positive association could disclose a role for OSA screening in patient with metabolic abnormalities, allowing an early recognition of the sleep disorder and preventing further severe consequences.

\section{Acknowledgement}

The authors have no acknowledgements.

\section{Conflict of Interest}

No conflict of interest are to declare.

\section{References}

1. Parish JM, Adam T, Facchiano L (2007) Relationship of Metabolic Syndrome and Obstructive Sleep Apnea. Journal of Clinical Sleep Medicine 3(5): 467-472.

2. Xu S, Wan Y, Xu M, Ming J, Xing Y, et al. (2015) The association between obstructive sleep apnea and metabolic syndrome: a systematic review and meta-analysis. BMC Pulmonary Medicine 15:105.

3. Bonsignore MR, Borel AL, Machan E, Grunstein R (2013) Sleep apnoea and metabolic dysfunction. European respiratory Review 22(129): 353364.

4. Lam JC, Mak JC, Ip MS (2012) Obesity, obstructive sleep apnea and metabolic syndrome. Respirology 17: 223-226. 
ISSN: 2574-1241

DOI: 10.26717/BJSTR.2020.24.00417

Adelina Anton. Biomed J Sci \& Tech Res

(C) (P) This work is licensed under Creative

Submission Link: https://biomedres.us/submit-manuscript.php

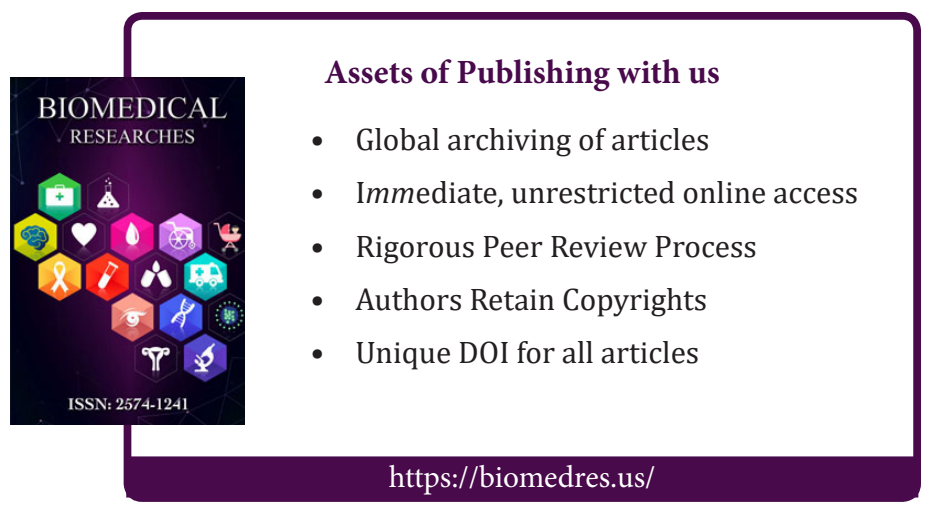

